\title{
Coeficiente de cultura do meloeiro irrigado com água salina estimado por modelo matemático
}

\author{
Crop coefficient of muskmelon irrigated with saline water estimated by mathematical model
}

\author{
Celsemy Eleutério Maia $^{\mathrm{I}^{*}}$ Elís Regina Costa de Morais ${ }^{\mathrm{I}}$
}

\section{RESUMO}

O coeficiente de cultura (Kc) do melão é importante para estimar a necessidade de água e para manejar eficientemente a irrigação. Com este trabalho objetivou-se avaliar um modelo matemático para estimar o Kc do meloeiro, em função dos dias após o plantio. O modelo matemático avaliado estimou, satisfatoriamente, os valores do coeficiente de cultura, porém as épocas de maior Kc não variaram com os tratamentos, mas alteraram os valores de Kc máximo e a taxa de crescimento absoluta máxima. Verificou-se também correlação positiva entre a época de Kc máximo com os valores de Kc máximo, a época da taxa de crescimento absoluta máxima e com a taxa de crescimento absoluta máxima, como também entre os valores de Kc máximo com a época de taxa de crescimento absoluto máximo e com a taxa de crescimento máxima, assim como entre a época da taxa de crescimento máxima e a taxa de crescimento absoluta máxima.

Palavras-chave: modelagem, salinidade, mulching.

\section{ABSTRACT}

The crop coefficient is necessary for estimating the water requirement in irrigation planning and management. This work aimed to evaluate a mathematical model to predict crop coefficient versus days after planting. The model provides a quite good performance in estimating the crop coefficient. The time in that maximum Kc occurred didn't vary with the treatments. However there had been alterations in values of maximum $\mathrm{Kc}$ and maximum absolute growth rate. Positive correlation was observed between the time for Kc maximum and Kc maximum, between time of maximum absolute growth rate and maximum absolute growth rate, as well as between $\mathrm{Kc}$ maximum and time of maximum absolute growth rate and maximum absolute growth rate, and between time of maximum absolute growth and maximum absolute growth rate.

Key words: modelling, salinity, mulching.

\section{INTRODUÇÃO}

A necessidade de utilização de água de qualidade inferior na irrigação é justificada, principalmente, pela escassez de áreas em que há água de boa qualidade. Esta realidade é verificada, sobretudo, em regiões semi-áridas, tornando conveniente o melhor manejo dessa água para diminuir os efeitos deletérios sobre o ambiente, em particular, em relação às culturas e ao solo.

A necessidade hídrica para determinada cultura é um dado básico que se deve conhecer para planejar e praticar o manejo adequado de qualquer projeto de irrigação. Dessa forma, torna-se oportuno o estudo de parâmetros que auxiliem na tomada de decisão. Um deles é o coeficiente de cultura (Kc), determinado pela razão entre a evapotranspiração máxima da cultura (ETc) e a evapotranspiração de referência (ETo). Este coeficiente depende do estádio de desenvolvimento da cultura, do sistema de irrigação, do arranjo de plantas e das condições meteorológicas reinantes (SOARES et al., 2001).

A salinidade do solo pode reduzir a evapotranspiração pela diminuição da disponibilidade de água para as plantas. Porém, em alguns casos, ocorre aumento da evaporação sob irrigação de alta freqüência na superfície do solo, compensando a diminuição do Kc pelo aumento da condutividade elétrica (CE) na zona radicular das plantas (ALLEN et al., 1998). MEDEIROS et al. (2003) observaram, também, alterações de Kc para melão irrigado com águas salinas e cobertura do solo, diminuindo não só com o aumento da salinidade da

\footnotetext{
'Departamento de Ciências Ambientais, Universidade Federal Rural do Semi-Árido, BR 110, km 47, CP 137, 59625-900, Mossoró,
} RN, Brasil. E-mail: celsemy@ufersa.edu.br.*Autor para correspondência. 
água de irrigação, mas também com o tipo de cobertura. Trabalhando com aplicação de água salina na cultura do pepino, BLANCO \& FOLEGATTI (2003) também observaram diminuição do coeficiente de cultura com o aumento da salinidade da água de irrigação.

Para ajustar o coeficiente de cultura em relação à época de plantio, geralmente se adota o ajuste dos pontos para as quatro etapas fenológicas e a curva é traçada definindo-se limites para cada uma delas, pela interseção das retas, estimada para cada uma delas (MEDEIROS et al., 2005). Alguns modelos matemáticos foram utilizados para estimar o coeficiente de cultura em função de dias após o plantio. Entre eles tem-se o modelo utilizado por BLANCO \& FOLEGATTI (2003), para determinação do Kc em pepino sob condições de ambiente protegido:

$$
\mathrm{Kc}=-0,024+\frac{1,547}{\left(1+\exp \left(\frac{-\mathrm{DAT}+52}{0,26131}\right)\right)^{0,0054}}
$$

Devido à simplicidade, equações polinomiais têm sido ajustadas por regressão, obtendose bons ajustes para o coeficiente de cultura em função da época de plantio (WRIGHT \& JANSEN, 1978). Modelos quadráticos e cúbicos foram ajustados por AYARS et al. (2003), enquanto MEDEIROS et al. (2001) ajustaram o modelo quadrático. O inconveniente desses modelos polinomiais é que representam apenas uma parte do ciclo da cultura, principalmente após o início do período de maior crescimento do Kc.
Tendo em vista o exposto, este trabalho teve como obter um modelo matemático para estimar o coeficiente de cultura (Kc) do meloeiro, em função de dias após o plantio (DAP), e avaliá-lo com dados de meloeiro irrigado com águas de diferentes salinidades e cobertura do solo.

\section{MATERIAL E MÉTODOS}

Para avaliação do modelo matemático, foram utilizados dados de Kc observado por MEDEIROS et al. (2003), (Tabela 1) a partir dos quais determinaram-se os coeficientes de cultura (Kc) para meloeiro irrigado com águas de diferentes salinidades, com e sem cobertura do solo. O ensaio foi conduzido entre dezembro de 2000 e fevereiro de 2001, na Fazenda São João Ltda, no município de Mossoró-RN (5¹2’ S, 37²12' W e 40m de altitude). O solo da área experimental é um Argissolo Vermelho Amarelo (MAIA, 2005).

O experimento foi conduzido com os tratamentos em esquema fatorial $3 \times 2 \times 2$, sendo três níveis de salinidade da água de irrigação $\left(\mathrm{S}_{1}=1,2 \mathrm{dS} \mathrm{m}^{-1}\right.$; $\left.\mathrm{S}_{2}=2,5 \mathrm{dS} \mathrm{m} \mathrm{m}^{-1} \mathrm{e} \mathrm{S}_{3}=4,4 \mathrm{dS} \mathrm{m} \mathrm{m}^{-1}\right)$, duas cultivares $\left(\mathrm{C}_{1}=\right.$ Trusty e $\mathrm{C}_{2}=$ Gold Mine) e duas condições de cobertura de solo $\left(\mathrm{M}_{1}=\right.$ sem e $\mathrm{M}_{2}=$ com cobertura de plástico dupla face - preto/prateado), totalizando 12 tratamentos: $\mathrm{T}_{1}=\mathrm{S}_{1} \mathrm{M}_{1} \mathrm{C}_{1}, \mathrm{~T}_{2}=\mathrm{S}_{1} \mathrm{M}_{1} \mathrm{C}_{2}, \mathrm{~T}_{3}=\mathrm{S}_{1} \mathrm{M}_{2} \mathrm{C}_{1}, \mathrm{~T}_{4}=\mathrm{S}_{1} \mathrm{M}_{2} \mathrm{C}_{2}$, $\mathrm{T}_{5}=\mathrm{S}_{2} \mathrm{M}_{1} \mathrm{C}_{1}, \mathrm{~T}_{6}^{2}=\mathrm{S}_{2} \mathrm{M}_{1} \mathrm{C}_{2}, \mathrm{~T}_{7}=\mathrm{S}_{2} \mathrm{MC}_{1}^{2}, \mathrm{~T}_{8}=\mathrm{S}_{2} \mathrm{M}_{2}^{2} \mathrm{C}_{2}$, $\mathrm{T}_{9}=\mathrm{S}_{3} \mathrm{M}_{1} \mathrm{C}_{1}, \mathrm{~T}_{10}=\mathrm{S}_{3} \mathrm{M}_{1} \mathrm{C}_{2}, \mathrm{~T}_{11}=\mathrm{S}_{3} \mathrm{M}_{2}^{2} \mathrm{C}_{1}, \mathrm{~T}_{12}=\mathrm{S}_{3} \mathrm{M}_{2} \mathrm{C}_{2}$. Utilizou-se delineamento experimental em blocos ao

Tabela 1 - Coeficiente de cultura (Kc) das cultivares de melão Trusty e Gold mine, irrigadas com água de diferentes salinidades e cultivadas sem cobertura de solo e com cobertura plástica sobre o solo, ao longo do ciclo (adaptado de MEDEIROS et al., 2003). Mossoró, 2003.

\begin{tabular}{|c|c|c|c|c|c|c|c|c|c|c|c|c|}
\hline \multirow{3}{*}{ 葛 } & \multicolumn{4}{|c|}{----------Salinidade 1----------- } & \multicolumn{4}{|c|}{----------Salinidade 2----------- } & \multicolumn{4}{|c|}{-----------Salinidade 3---------- } \\
\hline & \multicolumn{2}{|c|}{ M1 } & \multicolumn{2}{|c|}{ M2 } & \multicolumn{2}{|c|}{ M1 } & \multicolumn{2}{|c|}{ M2 } & \multicolumn{2}{|c|}{ M1 } & \multicolumn{2}{|c|}{ M2 } \\
\hline & $\mathrm{C} 1$ & $\mathrm{C} 2$ & $\mathrm{C} 1$ & $\mathrm{C} 2$ & $\mathrm{C} 1$ & $\mathrm{C} 2$ & $\mathrm{C} 1$ & $\mathrm{C} 2$ & $\mathrm{C} 1$ & $\mathrm{C} 2$ & C1 & $\mathrm{C} 2$ \\
\hline 1 & 0,24 & 0,27 & 0,17 & 0,20 & 0,25 & 0,27 & 0,18 & 0,20 & 0,22 & 0,25 & 0,15 & 0,18 \\
\hline 2 & 0,24 & 0,27 & 0,17 & 0,20 & 0,25 & 0,27 & 0,18 & 0,20 & 0,22 & 0,25 & 0,15 & 0,18 \\
\hline 3 & 0,24 & 0,27 & 0,17 & 0,20 & 0,25 & 0,27 & 0,18 & 0,20 & 0,23 & 0,25 & 0,15 & 0,18 \\
\hline 4 & 0,36 & 0,39 & 0,25 & 0,28 & 0,36 & 0,39 & 0,24 & 0,27 & 0,31 & 0,34 & 0,20 & 0,23 \\
\hline 5 & 0,59 & 0,56 & 0,48 & 0,46 & 0,56 & 0,54 & 0,46 & 0,44 & 0,51 & 0,49 & 0,41 & 0,38 \\
\hline 6 & 0,81 & 0,73 & 0,72 & 0,64 & 0,77 & 0,69 & 0,68 & 0,60 & 0,71 & 0,62 & 0,62 & 0,53 \\
\hline 7 & 0,99 & 0,83 & 0,85 & 0,69 & 0,97 & 0,81 & 0,84 & 0,68 & 0,87 & 0,71 & 0,74 & 0,58 \\
\hline 8 & 0,95 & 0,80 & 0,79 & 0,63 & 0,96 & 0,80 & 0,79 & 0,64 & 0,85 & 0,70 & 0,69 & 0,53 \\
\hline 9 & 0,78 & 0,65 & 0,65 & 0,52 & 0,76 & 0,63 & 0,62 & 0,49 & 0,67 & 0,54 & 0,54 & 0,41 \\
\hline 10 & 0,59 & 0,48 & 0,52 & 0,41 & 0,53 & 0,42 & 0,45 & 0,35 & 0,46 & 0,35 & 0,39 & 0,28 \\
\hline
\end{tabular}

${ }^{*}$ Tempo em semana.

Salinidade $1\left(\mathrm{CE}=1,2 \mathrm{dS} \mathrm{m}^{-1}\right)$, Salinidade $2\left(\mathrm{CE}=2,5 \mathrm{dS} \mathrm{m}^{-1}\right)$, Salinidade $3\left(\mathrm{CE}=4,4 \mathrm{dS} \mathrm{m}^{-1}\right)$.

M1 = sem cobertura, M2 = com cobertura.

C1 = melão Trusty, C2 = melão Gold Mine.

Ciência Rural, v.38, n.5, ago, 2008. 
acaso com quatro repetições, sendo as parcelas experimentais formadas por três fileiras de $6 \mathrm{~m}$ com 30 plantas por fileira. As duas águas de menor salinidade foram obtidas em poços do arenito Açu e do calcário Jandaíra água de maior salinidade foi obtida com a adição de $\mathrm{NaCl}$ à água do poço calcário, sendo aplicadas quando da irrigação por gotejamento.

O modelo proposto é dado por

$\mathrm{Kc}=\mathrm{Kc}_{\mathrm{ie}}+10^{\mathrm{a}+\mathrm{bt}+\mathrm{ct}^{2}}$, sendo Kc o coeficiente de cultura, $\mathrm{Kc}_{\mathrm{ie}}$ o coeficiente de cultura inicial estimado, t o tempo após o plantio (semanas) e $a, b$ e $c$ coeficientes do modelo. Os valores de $\mathrm{Kc}_{\mathrm{ie}}$, a, b e c foram ajustados por análise de regressão não-linear, pelo método dos mínimos quadrados.

$\mathrm{O}$ tempo em que se verifica o valor de Kc máximo (T.Kc $\left.\mathrm{max}_{\max }\right)$ durante o ciclo da cultura foi obtido por T.Kc $\mathrm{max}_{\max }=\frac{-\mathrm{b}}{2 \mathrm{c}}$.O valor de Kc máximo $\left(\mathrm{Kc}_{\max }\right)$ foi estimado por $K c_{\max }=K c_{i e}+\operatorname{ant} \log \left(a-\frac{b^{2}}{4 c}\right)$ A taxa de crescimento absoluto de Kc (TCAKc) foi calculada por

TCAKc $=10^{\mathrm{a}+\mathrm{bx}+\mathrm{cx}}{ }^{2} *(\mathrm{~b}+2 \mathrm{cx}) * \ln (10)$. $\mathrm{O}$ tempo em que ocorre a maior taxa de crescimento absoluta do Kc (T.TCA ${ }_{\max }$ ) foi estimado por

$$
\text { T.TCA }_{\max }=\frac{\sqrt{\frac{-2 c}{\ln (10)}}-b}{2 c}
$$

A taxa de crescimento máxima (TCA ${ }_{\text {max }}$ ) foi obtida substituindo-se T.TCA ${ }_{\text {Max }}$ na curva de TCAKc.
Para as características de $\mathrm{Kc}_{\text {max }}$, T.Kc $\mathrm{C}_{\text {max }}$,

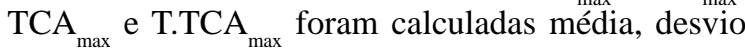
padrão e coeficiente de variação e, para as mesmas características, foi feita correlação.

\section{RESULTADOS E DISCUSSÃO}

O modelo proposto apresentou bom ajuste para todos os tratamentos, refletido pelos elevados coeficientes de determinação $\left(\mathrm{R}^{2}\right)$, sendo iguais ou superiores a 0,97 (Tabela 2 ). Os valores de $\mathrm{R}^{2}$ foram superiores aos obtidos por AL-JAMAL et al. (1999), em cebola, que utilizaram o modelo polinomial cúbico e o modelo AZSCHED, com Kc em função de graus-dia.

Comparando os valores dos parâmetros ajustados do modelo proposto (Tabela 2) com outros da literatura, verificou-se que HUNSAKER (1999), apesar de ter obtido bom ajuste de Kc em função de dias após o plantio utilizando o modelo de quinta ordem $\left(r^{2}=0,92\right)$, os valores de $\mathrm{Kc}_{\mathrm{ini}}$ estimados por este modelo foram de aproximadamente 0,11 , enquanto que, metodologia da FAO (ALLEN et al., 1998), foi de 0,23, ou seja, mais que o dobro, diferente dos valores estimados de $\mathrm{Kc}_{\text {ini }}$ pelo modelo proposto neste trabalho. Estes foram muito próximos do sugerido pela FAO e dos observados no campo por MEDEIROS et al. (2003). A relação entre os valores observados e os valores estimados pelo modelo, para todos os tratamentos e todas épocas de amostragem, observase que o intercepto e o coeficiente angular foram estatisticamente iguais a zero e um, respectivamente (Figura 1).

Tabela 2 - Valores dos coeficientes estimados do modelo proposto, coeficiente de determinação $\left(\mathrm{r}^{2}\right)$, tempo em que ocorre o Kc máximo $\left(T . K c_{M a x}\right)$, valor do $K c$ máximo $\left(K_{c_{\max }}\right)$, tempo em que ocorre a taxa de crescimento máxima (T.TCA $\left.A_{\max }\right)$ e a taxa de crescimento máxima do Kc (TCA max $)$. Mossoró, 2003.

\begin{tabular}{|c|c|c|c|c|c|c|c|c|c|c|c|c|}
\hline & \multicolumn{4}{|c|}{----------Salinidade 1---------- } & \multicolumn{4}{|c|}{----------Salinidade 2---------- } & \multicolumn{4}{|c|}{----------Salinidade 3---------- } \\
\hline & \multicolumn{2}{|c|}{ M1 } & \multicolumn{2}{|c|}{ M2 } & \multicolumn{2}{|c|}{ M1 } & \multicolumn{2}{|c|}{ M2 } & \multicolumn{2}{|c|}{ M1 } & \multicolumn{2}{|c|}{ M2 } \\
\hline & C1 & $\mathrm{C} 2$ & C1 & C2 & C1 & $\mathrm{C} 2$ & C1 & $\mathrm{C} 2$ & C1 & $\mathrm{C} 2$ & C1 & $\mathrm{C} 2$ \\
\hline$K c_{i e}$ & 0,21 & 0,25 & 0,14 & 0,18 & 0,24 & 0,26 & 0,16 & 0,19 & 0,21 & 0,24 & 0,13 & 0,17 \\
\hline $\mathrm{a}$ & $-3,20$ & $-3,27$ & $-3,14$ & $-3,19$ & $-3,73$ & $-3,66$ & $-3,71$ & $-3,75$ & $-3,74$ & $-3,87$ & $-3,68$ & $-4,00$ \\
\hline b & 0,83 & 0,84 & 0,80 & 0,80 & 0,97 & 0,94 & 0,96 & 0,96 & 0,96 & 0,99 & 0,94 & 1,02 \\
\hline C & $-0,06$ & $-0,06$ & $-0,05$ & $-0,06$ & $-0,06$ & $-0,06$ & $-0,06$ & $-0,07$ & $-0,07$ & $-0,07$ & $-0,06$ & $-0,07$ \\
\hline$r^{2}$ & 0,99 & 0,99 & 0,98 & 0,98 & 0,99 & 0,99 & 0,99 & 0,99 & 0,99 & 0,99 & 0,99 & 0,99 \\
\hline T.Kc $\mathrm{Cax}_{\text {Max }}$ & 7,47 & 7,28 & 7,49 & 7,25 & 7,46 & 7,26 & 7,42 & 7,20 & 7,41 & 7,18 & 7,38 & 7,11 \\
\hline $\mathrm{Kc}_{\text {Max }}$ & 0,99 & 0,84 & 0,85 & 0,70 & 0,99 & 0,83 & 0,84 & 0,69 & 0,89 & 0,73 & 0,75 & 0,59 \\
\hline T.TCA ${ }_{\text {Max }}$ & 5,49 & 5,33 & 5,47 & 5,27 & 5,63 & 5,43 & 5,59 & 5,39 & 5,58 & 5,41 & 5,53 & 5,37 \\
\hline TCA $_{\text {Max }}$ & 0,24 & 0,18 & 0,21 & 0,16 & 0,25 & 0,19 & 0,22 & 0,17 & 0,23 & 0,17 & 0,20 & 0,15 \\
\hline
\end{tabular}

Salinidade $1\left(\mathrm{CE}=1,2 \mathrm{dS} \mathrm{m}^{-1}\right)$, Salinidade $2\left(\mathrm{CE}=2,5 \mathrm{dS} \mathrm{m}^{-1}\right)$, Salinidade $3\left(\mathrm{CE}=4,4 \mathrm{dS} \mathrm{m}^{-1}\right)$.

M1 = sem cobertura, M2 = com cobertura.

C1 = melão Trusty, C2 = melão Gold Mine.

Ciência Rural, v.38, n.5, ago, 2008. 


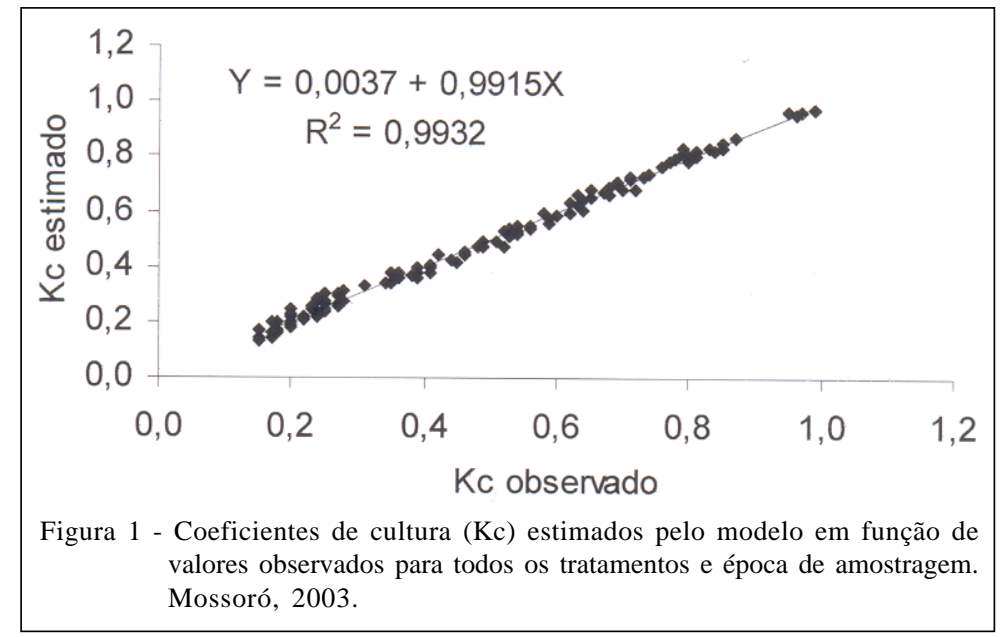

Avaliando-se os tratamentos pelo tempo para se obter o Kc máximo (T.Kc $\mathrm{Max}$ ), o Kc máximo $\left(\mathrm{Kc}_{\max }\right)$, o tempo para a taxa de crescimento absoluta máxima (T.TCA ${ }_{\max }$ ) e a taxa de crescimento absoluta máxima ( $\mathrm{TCA}_{\text {Max }}$ ) obtidos pelo modelo proposto, observa-se que T.Kc $\mathrm{Max}_{\text {ax }}$ acorreu aproximadamente 7,3 semanas após o plantio (51,3 dias) e que este, praticamente, foi semelhante para todos os tratamentos, indicado pelo valor do coeficiente de variação (CV) inferior a 2\%. O mesmo comportamento foi observado para o T.TCA $A_{\max }$ que, para todos os tratamentos, foi, em média, de 5,5 semanas após o plantio (38,2 dias), também com baixo valor de CV. Isso implica que os tratamentos (salinidade da água, cobertura do solo e cultivares) não influenciaram os valores de T.Kc ${ }_{\text {Max }} \mathrm{e}$ T.TCA $_{\max }$. Entretanto, os tratamentos influenciaram os valores de $\mathrm{Kc}_{\max }$ e a TCA $\mathrm{Max}_{\text {ax }}$, indicada pelos maiores valores do CV (Tabela 3). Avaliando os modelos polinomiais cúbicos, propostos por AZSCHED e FAO (ALLEN et al., 1998) em função dos graus-dia, ALJAMAL et al. (1999) verificaram que estes diferiram para $\mathrm{T.Kc}_{\max }$ e $\mathrm{Kc}_{\max }$, sendo que o modelo da FAO atinge $\mathrm{Kc}_{\max }$ antes que os modelos cúbicos e de AZSCHED. Para os valores de $\mathrm{Kc}_{\text {max }}$, o modelo da FAO

Tabela 3 - Valores médios, desvio padrão e coeficiente de variação (CV) para a época de Kc máximo (T.Kc $c_{\operatorname{Max}}$, semanas), valor de $\mathrm{Kc}$ máximo ( $\mathrm{Kc}_{\max }$ ), época da maior taxa de crescimento absoluta de Kc $\left(\right.$ T.TCA $_{\text {Max }}$, semana) e a taxa de crescimento absoluta máxima $\left(\mathrm{TCA}_{\mathrm{Max}}\right)$ para meloeiro os tratamentos. Mossoró, 2003.

\begin{tabular}{lccc}
\hline & Média & Desvio Padrão & CV (\%) \\
\hline T.KC $_{\text {Max }}$ & 7,33 & 0,1278 & 1,75 \\
$\mathrm{KC}_{\text {Max }}$ & 0,81 & 0,1204 & 14,92 \\
T.TCA $_{\max }$ & 5,46 & 0,1106 & 2,03 \\
$\mathrm{TCA}_{\max }$ & 0,20 & 0,0329 & 16,65 \\
\hline
\end{tabular}

estimou valores intermediários entre os modelos de AZSCHED e o modelo cúbico.

O maior valor de Kc foi observado para o melão Trusty cultivado sem cobertura do solo e irrigado com água de CE de 1,2dS m-1 (T1) e o menor para o melão Gold Mine, cultivado em solo coberto com plástico de polietileno prateado e irrigado com água de CE de 4,4dS $\mathrm{m}^{-1}$ (T12), sendo que a diminuição do Kc com o aumento da salinidade ocorreu em função da redução da evapotranspiração (ETP), conseqüentemente diminuindo a disponibilidade de água no solo para as plantas (ALLEN et al., 1998). A diminuição do Kc com o aumento da salinidade da água de irrigação e com a cobertura do solo também foi verificada por AL-JAMAL et al. (1999), trabalhando com cebola.

Avaliando-se a relação entre T.Kc ${ }_{\mathrm{Max}}, \mathrm{Kc}_{\max }$, T.TCA $_{\text {Max }}$ e TCA ${ }_{\text {Max }}$, observa-se correlação entre essas variáveis (Tabela 4). Assim sendo, quanto maior $\mathrm{T} \mathrm{KC}_{\text {Max }}$, maiores os valores de $\mathrm{Kc}_{\max }, \mathrm{T}_{\text {.TC }} \mathrm{e}$ $\mathrm{TCA}_{\mathrm{Max}}$. O mesmo comportamento foi verificado entre $\mathrm{KC}_{\text {Max }}$ com T.TC Max $_{\text {e TCA }}$, e entre T.TC Max $_{\text {Max }}$ TCA Max $_{\text {. }}$ Comparando-se valores de T.K. $\mathrm{Kc}_{\text {Max }}, \mathrm{Kc}_{\max }$, T.TCA $_{\text {Max }}$ e TCA ${ }_{\text {Max }}$, por tratamento, observa-se que a cultivar Gold Mine $\left(\mathrm{C}_{2}\right)$ apresentou menores valores, quando comparada com a Trusty $\left(\mathrm{C}_{1}\right)$ (Tabela 5). Para

Tabela 4 - Matriz de correlação entre época de Kc máximo (T.Kc $c_{\text {Max }}$, semana), valor de $\mathrm{Kc}$ máximo $\left(\mathrm{Kc}_{\max }\right)$, época da maior taxa de crescimento absoluta do Kc

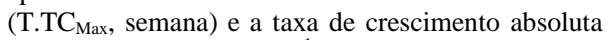
máxima (TCA ${ }_{\text {Max }}$, semana $\left.{ }^{-1}\right)$. Mossoró, 2003.

\begin{tabular}{lcccc}
\hline & T.Kc $_{\max }$ & $\mathrm{Kc}_{\max }$ & T.TCA $_{\max }$ & TCA $_{\max }$ \\
\hline${\mathrm{T} . K c_{\max }}$ & 1,00 & & & \\
$\mathrm{Kc}_{\max }$ & 0,84 & 1,00 & & \\
$\mathrm{~T}_{\mathrm{TCA}}$ & 0,73 & 0,64 & 1,00 & \\
$\mathrm{TCA}_{\max }$ & 0,93 & 0,92 & 0,86 & 1,00 \\
\hline
\end{tabular}

Todas as correlações foram significativas a 5\% de probabilidade.

Ciência Rural, v.38, n.5, ago, 2008. 
Tabela 5 - Valores médios para época de Kc máximo ( $\mathrm{T} . \mathrm{Kc}_{\mathrm{Max}}$, semana), valor de Kc máximo ( $\mathrm{Kc}_{\mathrm{max}}$ ), época da maior taxa de crescimento

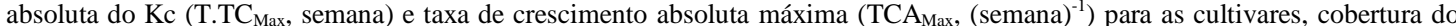
solo e salinidade da água de irrigação. Mossoró, 2003.

\begin{tabular}{|c|c|c|c|c|c|c|c|}
\hline & $\mathrm{C}_{1}$ & $\mathrm{C}_{2}$ & $\mathrm{M}_{1}$ & $\mathrm{M}_{2}$ & $\mathrm{~S}_{1}$ & $\mathrm{~S}_{2}$ & $\mathrm{~S}_{3}$ \\
\hline $\mathrm{T} . \mathrm{Kc}_{\max }$ & 7,44 & 7,21 & 7,34 & 7,31 & 7,37 & 7,34 & 7,27 \\
\hline $\mathrm{Kc}_{\max }$ & 0,88 & 0,73 & 0,88 & 0,74 & 0,85 & 0,84 & 0,74 \\
\hline T.TCA max $_{\text {m }}$ & 5,55 & 5,37 & 5,48 & 5,44 & 5,39 & 5,51 & 5,47 \\
\hline $\mathrm{TCA}_{\max }$ & 0,23 & 0,17 & 0,21 & 0,19 & 0,20 & 0,21 & 0,19 \\
\hline
\end{tabular}

Salinidade $1\left(\mathrm{CE}=1,2 \mathrm{dS} \mathrm{m}^{-1}\right)$, Salinidade $2\left(\mathrm{CE}=2,5 \mathrm{dS} \mathrm{m}^{-1}\right)$, Salinidade $3\left(\mathrm{CE}=4,4 \mathrm{dS} \mathrm{m}^{-1}\right)$.

M1 = sem cobertura, M2 = com cobertura.

C1 = melão Trusty, C2 = melão Gold Mine.

as coberturas, apenas os valores de $\mathrm{Kc}_{\text {max }}$ foram superiores para $M_{1}$, em comparação a $M_{2}$, enquanto que os valores de T.Kc $c_{\text {Max }}$, T.TCA $A_{\text {Max }}$ e TCA Max $_{\text {. }}$ praticamente não apresentaram diferença ente $M_{1}$ e $M_{2}$. Comparando-se o efeito da salinidade da água de irrigação, observa-se tendência de diminuição dos valores de T.Kc $c_{\text {Max }}$, Kc $c_{\max }$, e TCA ${ }_{\text {Max }}$ com o aumento da salinidade da água de irrigação, principalmente acima de 2,5dS $\mathrm{m}^{-1}$. Diminuição dos valores de Kc também pode ser observada durante todo o ciclo do meloeiro com aumento da salinidade da água acima de 2,5dS m ${ }^{-1}$ (Figura 2).

\section{CONCLUSÕES}

O modelo matemático avaliado estimou satisfatoriamente o coeficiente de cultura (Kc). Não houve influência da salinidade da água de irrigação e das cultivares para época de maior Kc, como também para a época de maior taxa de crescimento absoluto do Kc.

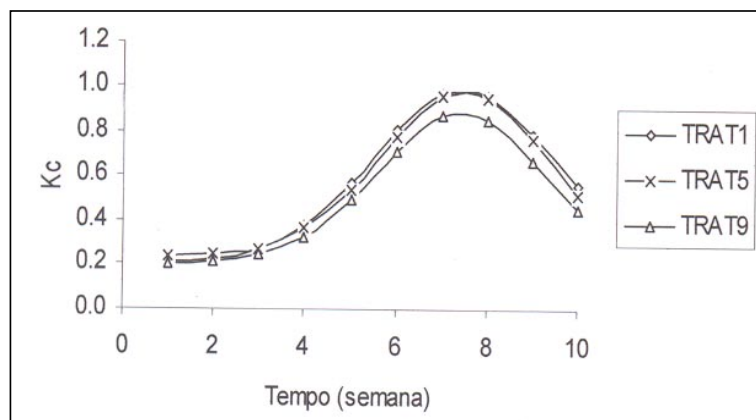

$\mathrm{C}_{1} \mathrm{M}_{1}$

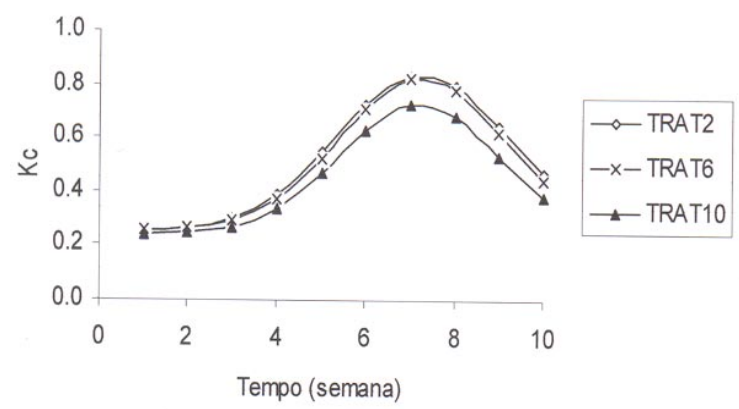

$\mathrm{C}_{2} \mathrm{M}_{1}$

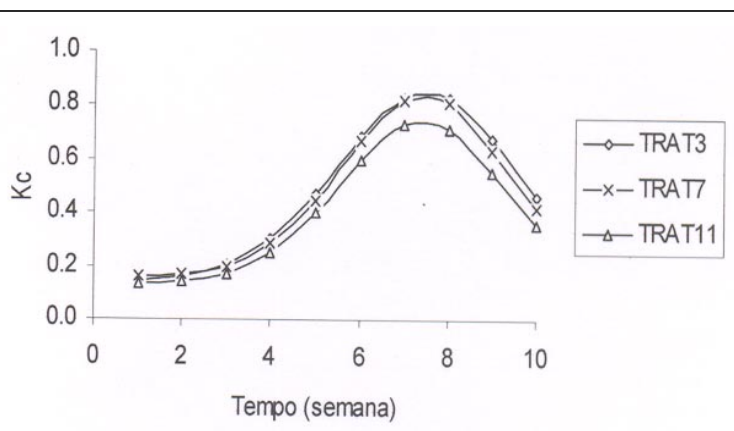

$\mathrm{C}_{1} \mathrm{M}_{2}$

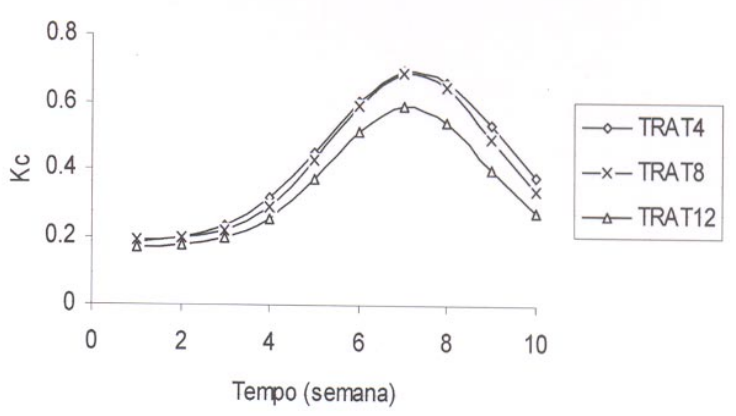

$\mathrm{C}_{2} \mathrm{M}_{2}$

Figura 2 - Coeficiente de cultura $(\mathrm{Kc})$ estimados ao longo do ciclo de duas cultivares de meloeiro (M1 = Trusty e M2 = Gold Mine), sem (M1) e com (M2) cobertura plástica sobre o solo. Mossoró, 2003.

Ciência Rural, v.38, n.5, ago, 2008. 
Entretanto, houve influência nos valores de Kc máximo e na taxa de crescimento absoluto máximo do Kc

\section{REFERÊNCIAS}

AL-JAMAL, M.S. et al. Yield-based, irrigated onion crop coefficients. Applied Engineering in Agriculture, St. Joseph, v.15, n.6, p.659-668, 1999.

ALLEN, R.G. et al. Crop evapotranspiration: guidelines for computing crop requirements. FAO irrigation and drainage. Rome: FAO, 1998. 300p. (Paper 24).

AYARS, J.E. et al. Water use by drip-irrigated late-season peaches. Irrigation Science, New York, v.22, p.187-194, 2003.

BLANCO, F.F.; FOLEGATTI, M.V. Evapotranspiration and crop coefficient of cucumber in greenhouse. Revista Brasileira de Engenharia Agrícola e Ambiental, Campina Grande, v.7, n.2, p.285-291, 2003.

HUNSAKER, D.J. Basal crop coefficients and water use for early maturity cotton. Transactions ASAE, St. Joseph, v.42, n.4, p.927-936, 1999.

MAIA, C.E. Modelos matemáticos para a estimativa da geometria do volume de bulbo molhado por irriogação por gotejamento superficial no agropolo Assu-Mossoró.
2005. 127f. Tese (Doutorado em Recursos Naturais) - Curso de Pós-graduação em Recursos Naturais, Universidade Federal de Campina Grande.

MEDEIROS, F.A.S.B. et al. Necessidade hídrica do meloeiro irrigado com água de diferentes salinidades e cultivado com ou sem cobertura do solo. Revista Brasileira de Engenharia Agrícola e Ambiental, Campina Grande, v.9, n.2, p.234238, 2005.

MEDEIROS, G.A. et al. The influence of crop canopy on evapotranspiration and crop coefficient on bean (Phaseolus vulgaris, L.). Agricultural Water Management, Amsterdam. v.34, n.3, p.211-224, 2001.

MEDEIROS, J.F. et al. Coeficiente de cultura do meloeiro irrigado com águas de diferentes salinidades e cultivado com e sem cobertura do solo. In: CONGRESSO NACIONAL DE IRRIGAÇÃO E DRENAGEM, 13., 2003, Juazeiro, BA. Resumos... Juazeiro: ABID, 2003. CD Rom.

SOARES, W.R. et al. Dependência do coeficiente de cultura no estádio inicial de desenvolvimento $\left(\mathrm{Kc}_{\mathrm{ini}}\right)$ à lâmina de irrigação e textura do solo. Revista Brasileira de Engenharia Agrícola e Ambiental, Campina Grande, v.5, n.1, p.23-27, 2001.

WRIGHT, J.L.; JENSEN, M.E. Development and evaluation of evapotranspiration models for irrigation scheduling. Transactions ASAE, St. Joseph, v.21, n.1, p.88-96, 1978. 